\title{
Conceptual content of the "healthy lifestyle" notion in the programs and activities for the formation of healthy lifestyle, carried out by teachers of preschool education in rural areas
}

\author{
Elena Egorova $^{1, *}$, Marina Kuznecova ${ }^{1}$ \\ ${ }^{1}$ Don State Technical University, 1, Gagarina sqr., 344003, Rostov-on-Don, Russia
}

\begin{abstract}
The purpose of the study is to study the content of rural teachers ' ideas PE a healthy lifestyle, which they broadcast in their work with children. The content analysis method is used to study 50 programs for the formation of a healthy lifestyle, which are being implemented in rural PE. The study showed that theoretical concepts more fully describe the "healthy lifestyle" notion, special attention is paid to the principles of its formation. Practical concepts are concentrated around human physiology, physical activity, sports, hygiene, and tempering. Axiological concepts receive a weak justification. The "healthy lifestyle" notion is revealed by rural teachers exclusively through the enumeration of its components. The unreliability of valeological information in the studied documents makes us doubt the competence of their authors. The nonfunctionality of the existing model of a healthy lifestyle requires additional research in the field of valeology, addressing the issue of the role of the worldview in this area, and strengthening the valeological and cultural aspects of teacher training. There is a need to strengthen the educational component aimed at forming ideas about the specifics of the lifestyle, culture and value system of rural and urban residents.
\end{abstract}

\section{Introduction}

The problem of this study arose on the basis of the assumption that in rural areas there are more opportunities and socio-cultural prerequisites for the implementation of HLS, since, in contrast to the urban environment, people here interact more directly with nature, the way of life in General is predetermined by more natural cycles and more close to the nature of human interests and needs. Many of the components that are traditionally included in HLS are immanently represented in rural life. children perceive and assimilate them directly from the activities of their environment.

In such circumstances, it can be assumed that the role of the teacher in the formation of a healthy lifestyle for rural children is to create a conceptual framework and a conscious attitude to their behavior, understanding the health benefits of living in rural areas. This is an important factor in the development of human resources in rural areas. In this regard, the

*Corresponding author: filonovaei@ya.ru 
question arose as to how conceptually complete are the knowledge and skills that PE teachers translate in the process of forming a healthy lifestyle for their students in rural areas.

Various educational programs for the formation of a healthy lifestyle in children, starting from preschool age, are the object of research interest both in Russia and abroad. However, the accents differ significantly. Russian scientists focus on the problems of physical education, sports and hygiene. Foreign authors are dominated by studies on healthy nutrition [1;2] and the formation of environmental thinking [3]. Even the problems of physical development of children are considered in the context of an ecological approach [4]. Another area of interest is teaching preschool children the basics of life safety [5].

It should also be noted that in Russian science, there is a detailed practice of preschool education in the field of HLS and research is being conducted on its effectiveness. And abroad, in particular in the United States, only the question is raised about the study of HLS and factors of its formation in preschoolers [6, p. 386]. For foreign researchers, HLS is not an indisputable value and necessity, since it is considered in the context of social, economic and political relations in a bourgeois-capitalist society, where it can become another factor of inequality [7].

However, there is a common feature that unites research in different countries: a significant discrepancy between theory and practice. Thus, at the theoretical level, Russian pedagogy is characterized by axiological, socio-cultural approaches, the approach of selfawareness and self-knowledge. In Western science, the motivational approach and the selfdetermination approach of Deci and Ryan are known, as well as the result-oriented approach of physical education by Jewett and others [8, p. 322-323]. We are also currently discussing an approach in which health is considered not as a result, but as a process, and educational programs for the formation of a healthy lifestyle are considered as continuous practices [9]. However, attempts to implement approaches in real working programs are always associated with certain technological problems.

According to independent monitoring, Russian senior preschoolers demonstrate a sufficient level of mental development to understand the basics of a healthy lifestyle. But at the same time, their ideas about HLS are one-sided, there is no conscious attitude to this phenomenon, there is no idea about cause-and-effect relationships in the health sphere. The basics of a healthy lifestyle are not fixed in behavioral models and habits: children know how to behave correctly in order to be healthy, but they do not do it. Foreign studies show that children learn the necessary habits and reproduce them in everyday life if the program contains elements aimed at forming practical skills of healthy behavior [1, p.176-178]. However, the approach of foreign colleagues is devoid of complexity and, as a rule, concerns any particular component of the HLS, for example, healthy nutrition.

It is also necessary to take into account research data showing that the ethno-cultural features that are most clearly manifested in the representatives of the rural population play a significant role in the formation of various models of HLS even within the framework of the General official culture $[10 ; 11]$. This means that the teacher is faced with the task of strengthening the positive aspects of socio-cultural influence and leveling the negative ones, as well as providing additional relevant knowledge. In this study, we tried to find out how the content of PE in rural areas corresponds to the designated task.

Thus, the purpose of the study is to identify and quantify the conceptual content of the "healthy lifestyle" notion in educational programs used by teachers in rural areas.

In the context of this study, the concept is understood within the framework of cultural (Yu. S. Stepanov) and linguistic-cultural (D. S. Likhachev) approaches as a semantic formation that characterizes the carriers of ethnic culture. The productivity of using the concept as an analysis tool is determined by the fact that it transforms the meaning of the word by the speaker's personal experience and the ethnocultural experience of the people. 
By the conceptual content of the notion, we understand the totality of those concepts that describe the notion under study in the understanding of the subject of speech, and the system of relationships and relationships between all these concepts.

\section{Materials and methods}

The research was conducted using the content analysis method. Content analysis is an integration of qualitative, meaningful and quantitative analysis of an array of homogeneous texts, during which semantic units, concepts, and images that are included in the subject field of research are identified, their content is determined, and frequency indicators are taken into account.

The materials for the study were 50 educational programs, event scenarios and guidelines on the topic "Healthy lifestyle" for pre-school educational institutions, developed and tested by teachers who conduct thir activities in rural areas. These materials were randomly selected among documents that are available on the Internet in the public domain. All works were performed by practicing teachers of preschool education not earlier than 2004.

In the course of the content analysis, basic concepts relatede to HLS were identified, the frequency of occurrence, the conceptual field covered by them, qualitative features and semantic nuances were evaluated.

The texts were also evaluated according to the following parameters:

- number and content of HLS concepts presented in the theoretical part of the text ("Introduction", "explanatory note", etc.) - concepts of the theory;

- the number and content of HLS concepts transmitted through practical programs (socio-cultural knowledge and practices that children learn in the course of educational activities) - concepts of practice;

- correspondence of practical and theoretical parts to each other;

- the degree of competence of teachers, correctness and accuracy of valeological information presented in their projects.

\section{Results}

\subsection{Identification of concepts}

Content analysis helped to identify in the studied texts two groups of concepts: the concepts of the theory, that is, those concepts that arise in the formulation of the theoretical assumptions of programs, scripts, guidelines; concepts practices, concepts, broadcast rural teachers through hands-on educational activities, which is reflected in the scenarios, lesson plans, etc.

The identified concepts were divided into five categories: the concept of HLS and health; Axiological concepts; concepts of knowledge and understanding; components, principles, factors of HLS; Principles of HLS formation. Depending on the context of the concept's appearance and the peculiarities of its presentation and meaning formation, similar or similar concepts in theory and practice were assigned to different categories.

Table 1 shows the concepts of the theory. 
Table 1. Concepts of the theory.

\begin{tabular}{|c|c|c|}
\hline № & Concept & Number of references \\
\hline \multicolumn{3}{|c|}{ The concept of healthy lifestyle and health } \\
\hline 1 & The formation of a healthy lifestyle & 40 \\
\hline 2 & Preservation of health & 30 \\
\hline 3 & Health as a resource & 14 \\
\hline 4 & Health - well-being, quality of life & 11 \\
\hline 5 & Health for the state & 6 \\
\hline 6 & Health - longevity & 1 \\
\hline \multicolumn{3}{|c|}{ Axiological concepts } \\
\hline 1 & Health as a value & 22 \\
\hline 2 & Health of society & 13 \\
\hline 3 & Value of life & 6 \\
\hline 4 & The body as the value & 2 \\
\hline 5 & Value of the individual & 2 \\
\hline 6 & The opposition of good and harm & 1 \\
\hline \multicolumn{3}{|c|}{ Concepts of knowledge and understanding } \\
\hline 1 & Awareness of cause-and-effect relationships & 9 \\
\hline 2 & Human body & 18 \\
\hline 3 & Ecology & 12 \\
\hline 4 & Cause of disease & 1 \\
\hline 5 & Illness as a need for help & 2 \\
\hline 6 & Right to health & 1 \\
\hline \multicolumn{3}{|c|}{ Components, principles, factors of healthy lifestyle } \\
\hline 1 & Physical activity/sports & 29 \\
\hline 2 & Food culture & 29 \\
\hline 3 & Hygiene & 28 \\
\hline 4 & Mode & 20 \\
\hline 5 & Moral / cultural behavior & 21 \\
\hline 6 & Hardening and massage & 20 \\
\hline 7 & Preventive control & 17 \\
\hline 8 & Prevention of deviations & 2 \\
\hline 9 & The role of medical care and medical services & 16 \\
\hline 10 & Impotence / uselessness of medicine & 4 \\
\hline 11 & Security & 16 \\
\hline 12 & Injuries & 3 \\
\hline 13 & Health-saving environment & 11 \\
\hline
\end{tabular}




\begin{tabular}{|l|l|c|}
\hline 14 & Giving up bad habits & 8 \\
\hline 15 & Rational behavior & 4 \\
\hline 16 & Healthy lifestyle and creativity & 2 \\
\hline 17 & Connection of health and emotions & 19 \\
\hline 18 & Psychological and mental health & 4 \\
\hline 19 & Social health & 1 \\
\hline 20 & Labor is a factor of social health & \multicolumn{2}{|c|}{ Principles of HLS formation } \\
\hline & \multicolumn{2}{|c|}{25} \\
\hline 1 & Healthy lifestyle practices & 22 \\
\hline 2 & $\begin{array}{l}\text { The principle of interaction between DOE teachers and family } \\
\text { / parents }\end{array}$ & 13 \\
\hline 3 & Awareness and activity & 12 \\
\hline 4 & Principle of systematization and systematization & 12 \\
\hline 5 & $\begin{array}{l}\text { The principle of focusing on the individual characteristics of } \\
\text { the child }\end{array}$ & 2 \\
\hline 6 & Gender approach in physical education & 9 \\
\hline 7 & The principle of immersion in HLS without declaring its basics & 18 \\
\hline 8 & The principle of informing & \\
\hline 9 & The principle of integrity and complexity & \\
\hline
\end{tabular}

Physical health does not appear as an independent concept. It is assumed that this is an obvious and basic component of health. Since the theoretical part of the documents is made "for adults", many concepts are deliberately not disclosed as self-evident.

Table 2 shows the concepts of the practice.

Table 2. Practice concepts.

\begin{tabular}{|l|l|c|}
\hline \multicolumn{1}{|c|}{ Concept } & \multicolumn{1}{|c|}{ The concept of healthy lifestyle and health } \\
\hline \multicolumn{2}{|c|}{ Number of references } \\
\hline 1 & Illness - a way to avoid unwanted activities & 1 \\
\hline 2 & Connection of health/illness with emotions & 17 \\
\hline 3 & Disease - lack of function & 16 \\
\hline 4 & Health/illness - quality of life & 8 \\
\hline 5 & Illness - need for help & 6 \\
\hline 6 & Health-beauty & 4 \\
\hline 7 & Health-strength & 3 \\
\hline 8 & Health - longevity & 2 \\
\hline 9 & Health-the future & 8 \\
\hline & & 18 \\
\hline 1 & Healthy lifestyle & Axiological concepts \\
\hline 2 & Health as a value & \\
\hline
\end{tabular}




\begin{tabular}{|l|l|l|}
3 & Children [a priori] don't like being sick & 9 \\
\hline 4 & Comparative value of health & 1 \\
\hline 5 & Weak explanation of the value/necessity of health & 6 \\
\hline 6 & Value of life & 2 \\
\hline 7 & Diversity and variability [of the human body] & 8 \\
\hline 8 & Harmful and useful/good and bad & 1 \\
\hline 9 & Relative harm and benefit & 2 \\
\hline 10 & The body as the value & 1 \\
\hline 11 & Person/Personality as a value & \\
\hline
\end{tabular}

Concepts of knowledge and understanding

\begin{tabular}{|l|l|c|}
\hline 1 & The essence and purpose of health & 7 \\
\hline 2 & Body and health & 25 \\
\hline 3 & Influence of the environment & 14 \\
\hline 4 & Microbes and viruses & 14 \\
\hline 5 & Ecology & 9 \\
\hline 6 & Injuries & 7 \\
\hline 7 & Treatment & 4 \\
\hline 8 & Life & 2 \\
\hline
\end{tabular}

Components, principles, factors of healthy lifestyle

\begin{tabular}{|l|l|c|}
\hline 1 & Conservation/health care & 15 \\
\hline 2 & Connection between sport and health & 42 \\
\hline 3 & Relationship between nutrition and health & 31 \\
\hline 4 & The link between health and hygiene & 30 \\
\hline 5 & Massage and tempering & 28 \\
\hline 6 & The importance of medicine & 25 \\
\hline 7 & Fear of doctors & 17 \\
\hline 8 & Health relationship with the regime & 18 \\
\hline 9 & Security & 9 \\
\hline 10 & Prevention & 6 \\
\hline 11 & Vaccinations & 6 \\
\hline 12 & Bad habits [as an object of struggle] & 4 \\
\hline 13 & Culture and morality & 1 \\
\hline 14 & Impotence/lack of medical care & \\
\hline & \multicolumn{2}{|l}{ Principles of HLS formation } \\
\hline 1 & The need for systematic training & \\
\hline
\end{tabular}


In the organization of practical classes, rural teachers pay SPECIAL attention to the formation of children's value attitude to health and consolidation of knowledge about the components of HLS, as well as the assimilation of appropriate practices.

\subsection{Assessment of compliance of practice and theory}

When comparing the concepts of theory and practice, there are significant differences in several criteria. A discrepancy in the number of concepts was revealed. In General, more theoretical concepts have been identified than practical ones. There is a slight predominance of practice concepts in the categories "Concepts", "Axiological concepts", "Knowledge and understanding". That is, teachers make significant efforts to explain, justify values, and form an understanding of healthy lifestyles in children. But the most striking differences are found in the category "Components, principles, factors", which presents the content of the HLS components. If in theory there are 20 of them, in practice there are only 14, that is, the theoretically stated components, principles and factors are not presented in any way in the practical classes (see table 3 ).

Table 3. Quantitative ratio of theoretical concepts and practice concepts by category.

\begin{tabular}{|c|c|c|}
\hline Categories & $\begin{array}{l}\text { Number of } \\
\text { theoretical concepts }\end{array}$ & $\begin{array}{l}\text { Number of practice } \\
\text { concepts }\end{array}$ \\
\hline The concept of healthy lifestyle and health & 6 & 9 \\
\hline Axiological concepts & 6 & 11 \\
\hline Concepts of knowledge and understanding & 6 & 8 \\
\hline $\begin{array}{l}\text { Components, principles, factors of healthy } \\
\text { lifestyle }\end{array}$ & 20 & 14 \\
\hline Principles of HLS formation & 9 & 1 \\
\hline Total: & 47 & 43 \\
\hline
\end{tabular}

Only in the ten documents studied, practical developments can be considered as corresponding to the stated theoretical grounds. In all other cases (40), the degree of correspondence between the concepts of practice and the concepts of theory fluctuates significantly, falling to $20 \%$. 


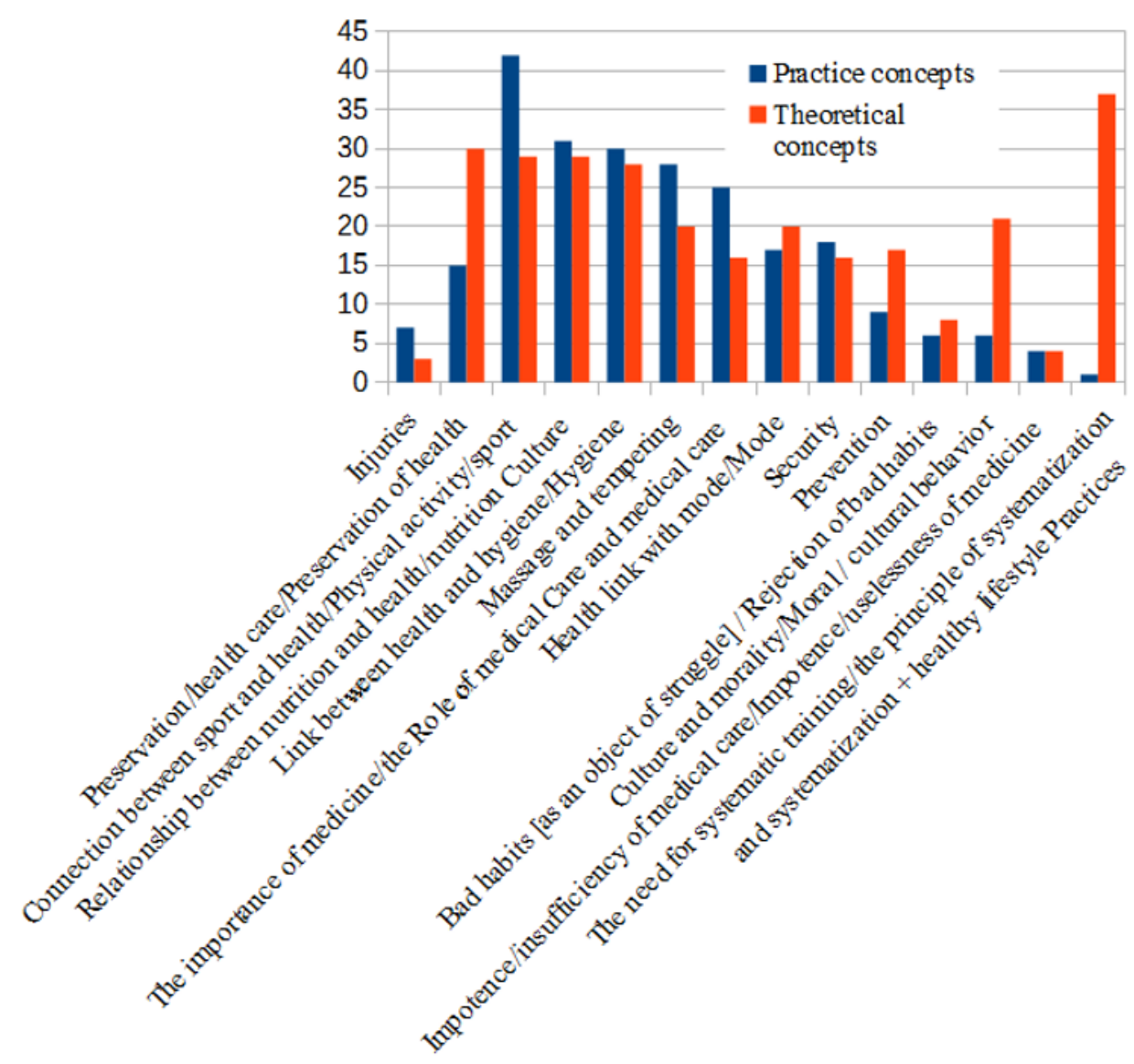

Fig. 1. Ratio of the number of mentions of concepts.

This situation is illustrated in Figure 1 and 2: here is the ratio of the number of mentions for those concepts that coincided (including partially).

Many concepts of practice are used as a tool to convey the meaning, value, rational and later behavioral consolidation of the basics of HLS. It is the instrumental role that explains their absence in the theory.

But there are concepts that are of fundamental importance in the formation of a healthy lifestyle, reflected in theory, but not represented in practice: health for the state, public health, awareness of cause and effect, social health, work-a factor of social health, rational behavior, psychological and mental health, awareness and activity. 


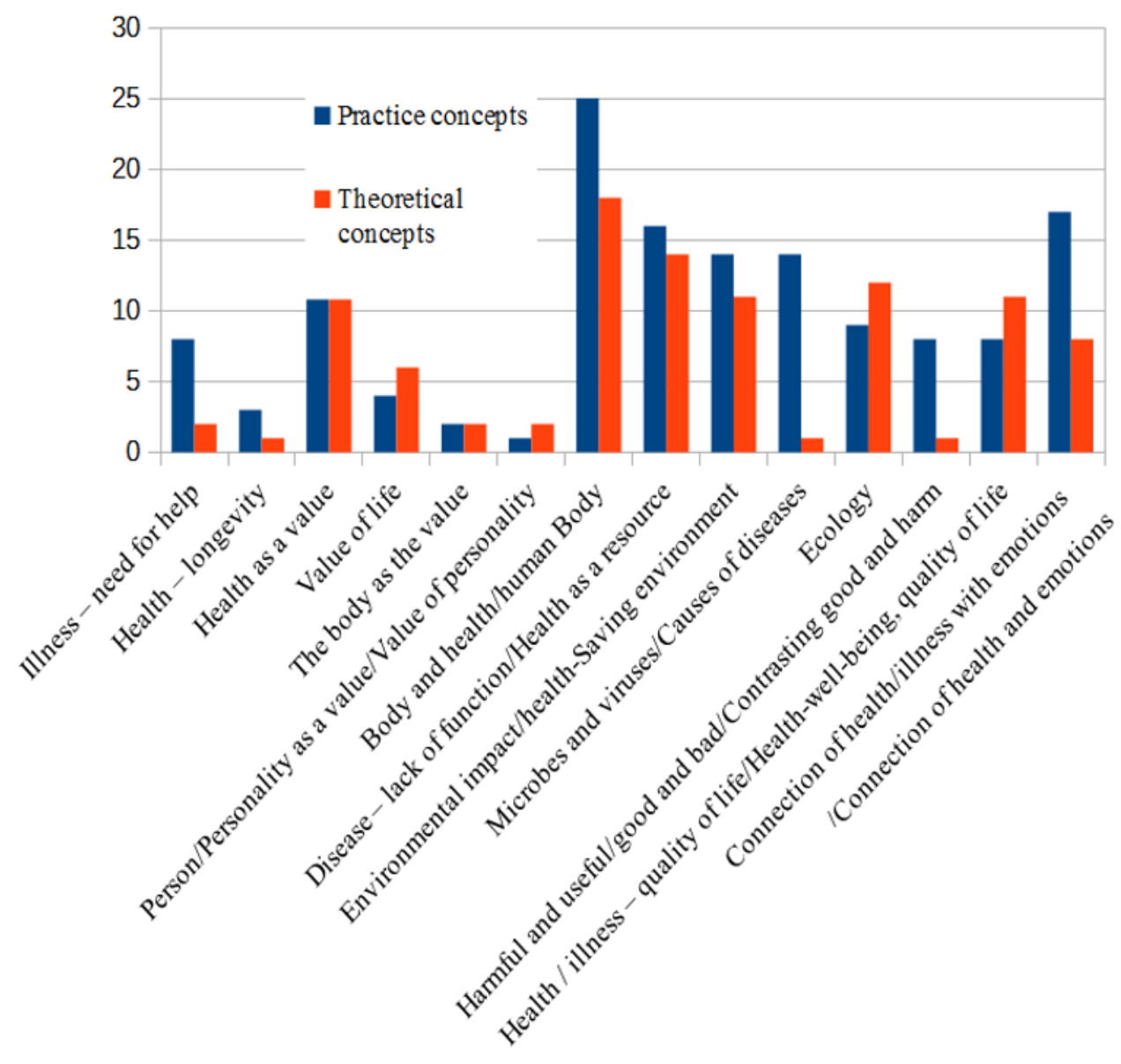

Fig. 2. Ratio of the number of mentions of concepts.

\subsection{Assessment of teachers ' competence}

In contrast to foreign teachers who do not feel competent in the work of forming a healthy lifestyle, they feel the need for special medical education for this [12], domestic specialists consider themselves accomplished professionals. However, the professionalism and competence of the authors of a significant number of documents studied raise some doubts: 24 of them contain false information and use questionable methods. There is an incorrect use of terminology, inadequate analogies and descriptions of physiological processes. 13 papers contain harmful or dangerous recommendations that are presented as useful.

\section{Discussion}

The authors of the studied materials demonstrate a steady interest in the topic of healthy nutrition. But it is understood almost exclusively as containing vitamins. The practice is skewed towards physical exercises. The assessment of the sport is extremely positive. No attention is paid to other types of physical activity, in particular - labor, which is of fundamental importance for rural areas. 
The emphasis in many programs is on skills, forming useful habits, and questions about the value and meaning of health, the essence of HLS are omitted, are not discussed with children and are not introduced into their cultural background in any way. Rural teachers are afraid to let children feel what the disease is in their play activities, to make it clear that this is a significant restriction of opportunities and a decrease in the quality of life. But without this understanding, it is difficult to understand the value of health. The concept of health appears less frequently than the concept of disease. At the same time, the concept of the disease often sounds first.

In the materials studied, there is no flexibility of broadcast settings: strict rules are given without the possibility of amendments to changing conditions and unique situations.

The theory does not provide a conceptual basis for the "healthy lifestyle" notion, which causes a lack of understanding of how to convey its meaning to students.

In many documents, the healthy lifestyle is identical to health care, which corresponds to the change of the paradigm of fighting the disease with the paradigm of preserving health [13].

No work refers to the worldview as the basis or component of a healthy lifestyle. All documents contain a weak axiology. In fact, we are dealing with a situation described by Jonathan Steffen: "We are regularly advised to do less of this or more of that in order to have more years of healthy life. In the essentially secular, consumer societies within which Western medicine operates, however, little mention is made of what we should, or could, be doing with those years" [14, p. 15]. But no educational effort will have a sustainable result if the child does not form a belief that health is really important to him.

In the studied programs, teachers, not parents, play a leading educational role. However, surveys of parents show that they leave the priority in forming a healthy lifestyle behind them. There is a conflict between the status of participants in the educational process.

The "healthy lifestyle" notion reconstructed in the theory corresponds to the integral normative model described by R. A. Kazimov [15]. But in practice, teachers broadcast a different model that is currently common in everyday consciousness: tautology is used to explain HLS ("this is health"); sports and proper nutrition are the main components of HLS, as well as the rejection of everything that is harmful to health; very rarely is a clear explanation of the meaning of health (health as a resource, health as a quality of life).

\section{Conclusions}

At the General theoretical level, we have to admit that the generally accepted model of HLS in Russian pedagogy is insufficient: there is no culture of health in it. But, as rightly noted by E. Bryndin and I. Bryndina, a full-fledged healthy lifestyle is possible only when the spiritual component and corresponding practices are activated at the level of the entire society [16, p. 67]. This area is in dire need of a worldview Foundation.

At the level of scientific knowledge, this means the need for socio-cultural studies of HLS, especially the basics of forming an appropriate worldview and axiology. In particular, rural educators should look for convincing and functional grounds for HLS in the way of life of people working on the land. It is necessary to use more productively the values that are already present in the culture surrounding the child, rather than trying to educate him in the spirit of abstract universal values.

At the level of pedagogical practice, the main conclusion is that changes are needed in the system of teacher training: strengthening of valeological components, expanding sociocultural competence and understanding of the specifics of the regions and settlements in which they carry out pedagogical activities. 


\section{References}

1. A. de Villiers et al., Ethnicity \& Disease 26(2), 171-180 (2016) DOI: 10.18865/ed.26.2.171

2. E. Oljans, H. Elmståhl, Y.M. Sydner, K. Hjälmeskog, Pedagogy, Culture \& Society 26(1), 35-49 (2018) https://doi.org/10.1080/14681366.2017.1345974

3. J. Merewether, Pedagogy, Culture \& Society 27(2), 233-250 (2019) https://doi.org/10.1080/14681366.2018.1460617

4. I. Renshaw, J-Y. Chow, Physical Education and Sport Pedagogy 24(2), 103-116 (2019) https://doi.org/10.1080/17408989.2018.1552676

5. K. Proulxa, F. Aboud, International Journal of Educational Development 66, 1-7 (2019) https://doi.org/10.1016/j.ijedudev.2019.01.007

6. S. Mollborn, L. James-Hawkins, E. Lawrence, P. Fomby, Journal of Health and Social Behavior 55(4), 386-402 (2014) DOI: https://doi.org/10.1177/0022146514555981.

7. W.K. Mariner, American Journal of Law \& Medicine 42, 284-309 (2016) DOI: https://doi.org/10.1177/0098858816658268

8. L. Haerens et al., Quest 63, 321-338 (2011)

9. M. Quennerstedt, Physical Education and Sport Pedagogy 24(1), 1-15 (2019) https://doi.org/10.1080/17408989.2018.1539705

10. N.S. Landale1 et al., Demographic Research 28(44), 1302-1338 (2013) DOI:10.4054/DemRes.2013.28.44

11. A.C. Lindsay, J.A. Salkeld, M.L. Greaney, F.D. Sands, Journal of Obesity 9 (2015) DOI: $10.1155 / 2015 / 409742$

12. N.Y.I. Cheng, M.Y.E. Wong, Health 7, 119-126 (2015) DOI: 10.4236/health.2015.71014

13. H. Siswanto, Proceedings International Seminar and Workshop on Public Health Action 1-2, 102-110 (2015)

14. S.J. Healthy, Lifestyle Genomics 11, 13-15 (2018) DOI: https://doi.org/10.1159/000487612

15. R.A. Kasimov, Economic and social changes: facts, trends, forecast 2(38), 161-172 (2015) DOI: 10.15838/esc/2015.2.38.10

16. E. Bryndin, I. Bryndina, International Journal of Psychological and Brain Sciences 2(3), 63-68 (2017) DOI: 10.11648/j.ijpbs.20170203.11 